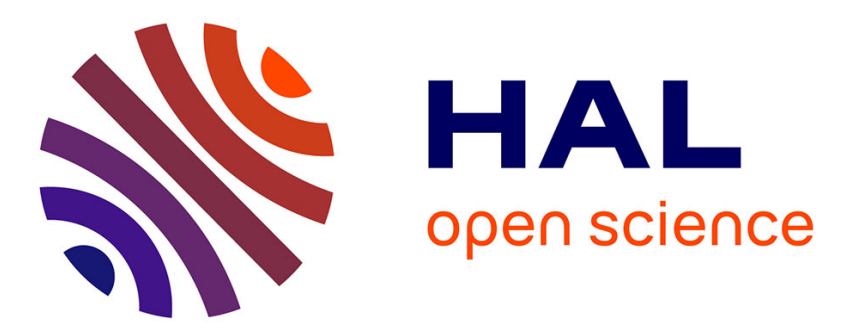

\title{
State-generating matrix for differential Mueller matrices of depolarizing optical media
}

\author{
Vincent Devlaminck, Jean-Michel M Charbois
}

\section{To cite this version:}

Vincent Devlaminck, Jean-Michel M Charbois. State-generating matrix for differential Mueller matrices of depolarizing optical media. Journal of the Optical Society of America. A Optics, Image Science, and Vision, 2020, 37 (6), pp.988-994. hal-02591505

\section{HAL Id: hal-02591505 \\ https://hal.science/hal-02591505}

Submitted on 15 May 2020

HAL is a multi-disciplinary open access archive for the deposit and dissemination of scientific research documents, whether they are published or not. The documents may come from teaching and research institutions in France or abroad, or from public or private research centers.
L'archive ouverte pluridisciplinaire HAL, est destinée au dépôt et à la diffusion de documents scientifiques de niveau recherche, publiés ou non, émanant des établissements d'enseignement et de recherche français ou étrangers, des laboratoires publics ou privés. 


\title{
State-generating Matrix for differential Mueller matrices of depolarizing optical media
}

\author{
V. Devlaminck* , J.M. Charbois \\ Université de Lille, CRIStAL, UMR 9189, 59650 Villeneuve d'Ascq, France \\ *Corresponding author: vincent.devlaminck@univ-lille.fr
}

\section{INTRODUCTION}

It is now well known [1-3] that a medium whose optical properties are functions of the thickness $z$ of the traversed medium, are governed by a differential equation:

$$
\frac{d \boldsymbol{M}(z)}{d z}=\boldsymbol{m}(z) \cdot \boldsymbol{M}(z)
$$

relating the Mueller matrix $\boldsymbol{M}(z)$ at a distance $z$ into the medium to its spatial derivative along the light propagation. For a non-depolarizing medium, this equation is completely defined by the knowledge of the initial condition and the differential Mueller matrix $\boldsymbol{m}(z)$ since this matrix is a deterministic one.

This differential matrix (noted $\boldsymbol{m}_{\mathrm{ND}}(z)$ in this case) can be written using 6 parameters $[4,5]$. According to the notation convention used, the parameters occupy different positions in the matrix. It is worth noticing that the matrix previously described in [4] used the standard quantum notation rather than the usual optical notation adopted in [5] and in this paper. With this convention:

$$
\boldsymbol{m}_{N D}(z)=\left[\begin{array}{cccc}
0 & -L D & -L D^{\prime} & C D \\
-L D & 0 & C B & L B^{\prime} \\
-L D^{\prime} & -C B & 0 & -L B \\
C D & -L B^{\prime} & L B & 0
\end{array}\right]
$$

where linear dichroism $(L D)$ and birefringence $(L B), 45^{\circ}$-linear dichroism $\left(L D^{\prime}\right)$ and birefringence $\left(L B^{\prime}\right)$, and circular dichroism $(C D)$ and birefringence $(C B)$ are the six phenomenological elementary polarization properties fully characterizing the medium. Generally these parameters are functions of the thickness $z$ of the traversed medium. The most elementary situation is given for homogeneous medium where these parameters are proportional to $z$.

The relations between these parameters (but also between the different notations used by the first authors on this subject [1,2,6]) and the corresponding physical effects are given in [7] for instance.

The underlying structure of this differential matrix is related to the 6 generators of the Lie algebra of $S O(3,1)($ the proper orthochronous Lorentz group) that can be dissociated in terms of more familiar quantities: 3 rotation generators $\boldsymbol{J}_{1}, \boldsymbol{J}_{2}, \boldsymbol{J}_{3}$ and 3 boost generators $\boldsymbol{K}_{1}, \boldsymbol{K}_{2}$ and $\boldsymbol{K}_{3}$ (these generators and their relations are described in Appendix $A$ of [8]):

$$
\boldsymbol{m}_{N D}(z)=-L D(z) \cdot \boldsymbol{K}_{1}-L D^{\prime}(z) \cdot \boldsymbol{K}_{2}+C D(z) \cdot \boldsymbol{K}_{3}+L B(z) \cdot \boldsymbol{J}_{1}+L B^{\prime}(z) \cdot \boldsymbol{J}_{2}-C B(z) \cdot \boldsymbol{J}_{3}
$$

The Lie algebra of the Lorentz group can be reduced to the direct product of two sub-algebras (the algebra of $S U(2)$, the group of two-dimensional unitary matrices with unit determinant) by transforming its generators $\left\{\boldsymbol{J}_{1}, \boldsymbol{J}_{2}, \boldsymbol{J}_{3}, \boldsymbol{K}_{1}, \boldsymbol{K}_{2}, \boldsymbol{K}_{3}\right\}$ to a new basis [9]: 


$$
\left\{\begin{array}{l}
\boldsymbol{M}_{m}=\frac{\boldsymbol{K}_{m}+i \boldsymbol{J}_{m}}{\sqrt{2}} \\
\boldsymbol{N}_{m}=\frac{\boldsymbol{K}_{m}-i \boldsymbol{J}_{m}}{\sqrt{2}}
\end{array} \text { with } m=1,2,3\right.
$$

The factor $\sqrt{2}$ is used in order to define some unitary transformations that will be necessary above. A straightforward calculation shows that: $\left[\boldsymbol{M}_{m}, \boldsymbol{N}_{n}\right]=0$ where $[\mathbf{A}, \mathbf{B}]=\mathbf{A B}-\mathbf{B A}$ is the commutator operator. This property of commutativity is not verified for $J$ and $K$ generators. It is also obvious that $\boldsymbol{N}_{m}=\boldsymbol{M}_{m}^{*}$.

It is useful to define a general retardation for each of the birefringence-dichroism pairs (usually referred to as spectroscopic notations); thus:

$$
\left\{\begin{array}{c}
L=-(L D+i L B) \\
L^{\prime}=-\left(L D^{\prime}+i L B^{\prime}\right) \\
C=C D+i C B
\end{array}\right.
$$

With these notations, Eq. (3) becomes:

$$
\boldsymbol{m}_{N D}(z)=\frac{L}{\sqrt{2}} \boldsymbol{M}_{1}+\frac{L^{\prime}}{\sqrt{2}} \boldsymbol{M}_{2}+\frac{C}{\sqrt{2}} \boldsymbol{M}_{3}+\frac{L^{*}}{\sqrt{2}} \boldsymbol{N}_{1}+\frac{L^{\prime *}}{\sqrt{2}} \boldsymbol{N}_{2}+\frac{C^{*}}{\sqrt{2}} \boldsymbol{N}_{3}
$$

Where * stands for the complex conjugate.

Eq. (6) is exactly the Eqs. (62) and (63) in the paper of Sheppard [10] where the expression of $\boldsymbol{m}(z)$ is derived from the Dirac matrices (termed $\Gamma_{\mathrm{ij}}$ in [10]) and can be related - at least in the case of homogeneous medium - to the $\boldsymbol{Z}$ matrix recently introduced by Kuntman [11]. The factor $1 / \sqrt{2}$ in Eq. (6) is necessary taking into account the factor $1 / \sqrt{2}$ in the definition of the matrices $\boldsymbol{M}_{\mathrm{m}}$ and $\boldsymbol{N}_{\mathrm{m}}$ given Eq. (4). The product of these two factors gives the present factor $1 / 2$ in the $\boldsymbol{\Gamma}_{\mathrm{ij}}$ matrices (Fig. 1 in Sheppard [10]).

\section{DEPOLARIZING MEDIUM}

\subsection{Depolarizing differential Mueller matrix}

For depolarizing medium the differential Mueller matrix must be considered [8, 12-13] as a matrix of random processes generated by the spatial fluctuations of the birefringence and dichroism components.

Eq. (1) is then a stochastic differential equation. This equation is now ccompletely defined by the knowledge of the initial condition (which can be random in the most general case) and the statistical laws associated to the differential matrix $\boldsymbol{m}(z)$

From a statistical point of view, Eq. (6) shows that we have to deal with three complex random processes $\left(L, L^{\prime}, C\right)$ and it is well known [14] that with such processes both the complex process and its conjugate must be considered in order to extract all the statistical information. It is obvious when we notice that $L D=-\left(L+L^{*}\right) / 2$ and $L B=-(L-$ $\left.L^{*}\right) / 2 i$ for instance.

$\boldsymbol{M}(z)$ is then seen as an average value of non-depolarizing Mueller matrices $\boldsymbol{M}_{\mathrm{ND}}(z)$ associated to their differential matrices $\boldsymbol{m}_{\mathrm{ND}}(z)$ but random $[12,15]$ :

$$
\boldsymbol{M}(z)=\left\langle\overleftarrow{Z} \exp \left(\int_{0}^{z} \boldsymbol{m}_{N D}(s) d s\right)\right\rangle=\overleftarrow{Z} \exp \left(\sum_{n=1}^{+\infty} \int_{0}^{z} \boldsymbol{c}^{n}(s) d s\right)
$$


The mean value is denoted by angular brackets, $\mathbf{c}^{(\mathrm{n})}$ stands for the cumulant of $n$ order and $\overleftarrow{Z}$ is a space-ordering operator introduced by Fox [16] that causes the factors in a product to appear in the same sequence as in the medium of interest. This space-ordering operator is made necessary since the matrices $\boldsymbol{m}_{\mathrm{ND}}\left(z_{1}\right)$ and $\boldsymbol{m}_{\mathrm{ND}}\left(z_{2}\right)$ do not commute for $z_{1} \neq$ $z_{2}$ in general.

\subsection{Complex random processes}

Since we are dealing with three complex random processes $\left(L, L^{\prime}, C\right)$, we introduce the complex $6 \times 6$ matrix $[17,18]$ in order to build complex vectors:

$$
\boldsymbol{T}_{6}=\frac{1}{\sqrt{2}}\left[\begin{array}{ll}
\boldsymbol{I}_{3} & -i \boldsymbol{I}_{3} \\
\boldsymbol{I}_{3} & +i \boldsymbol{I}_{3}
\end{array}\right]
$$

where $\boldsymbol{I}_{3}$ is the identity matrix of dimension 3. It obvious that $\boldsymbol{T}_{6}$ is Hermitian (meaning $\boldsymbol{T}_{6} \boldsymbol{T}_{6}^{H}=\boldsymbol{I}_{6}=\boldsymbol{T}_{6}^{H} \boldsymbol{T}_{6}$ where $H$ stands for the Hermitian conjugate).

From the real and imaginary parts of $\left(L, L^{\prime}, C\right)$, we define a vector of dimension $6 \times 1$ :

$$
\boldsymbol{r}=\left[\begin{array}{ll}
\boldsymbol{D}^{T} & B^{T}
\end{array}\right]^{T}=\left[\begin{array}{l}
\boldsymbol{D} \\
\boldsymbol{B}
\end{array}\right]
$$

With:

$$
\boldsymbol{D}=\left[\begin{array}{c}
-L D \\
-L D^{\prime} \\
C D
\end{array}\right] \quad \boldsymbol{B}=\left[\begin{array}{c}
-L B \\
-L B^{\prime} \\
C B
\end{array}\right]
$$

We also build two vectors of dimension $1 \mathrm{x} 3$ where $\boldsymbol{K}_{\mathrm{m}}$ and $\boldsymbol{J}_{\mathrm{n}}\left(\boldsymbol{M}_{\mathrm{m}}\right.$ and $\boldsymbol{N}_{\mathrm{n}}$ respectively $)$ are considered as the components of $\boldsymbol{K}$ and $\boldsymbol{J}(\boldsymbol{M}$ and $\boldsymbol{N}$ respectively) vectors:

$$
\begin{gathered}
\boldsymbol{K}=\left[\begin{array}{lll}
\boldsymbol{K}_{1} & \boldsymbol{K}_{2} & \boldsymbol{K}_{3}
\end{array}\right] \quad \boldsymbol{J}=\left[\begin{array}{lll}
\boldsymbol{J}_{1} & \boldsymbol{J}_{2} & \boldsymbol{J}_{3}
\end{array}\right] \\
\boldsymbol{M}=\left[\begin{array}{lll}
\boldsymbol{M}_{1} & \boldsymbol{M}_{2} & \boldsymbol{M}_{3}
\end{array}\right] \quad \boldsymbol{M}^{*}=\boldsymbol{N}=\left[\begin{array}{lll}
\boldsymbol{N}_{1} & \boldsymbol{N}_{2} & \boldsymbol{N}_{3}
\end{array}\right]
\end{gathered}
$$

From Eq. (3) and with these previously introduced notations, $\boldsymbol{m}_{\mathrm{ND}}(z)$ is given by:

$$
\boldsymbol{m}_{N D}(z)=\left[\begin{array}{ll}
\boldsymbol{K} & \boldsymbol{J}
\end{array}\right] \cdot\left[\begin{array}{l}
\boldsymbol{D} \\
\boldsymbol{B}
\end{array}\right]=\left[\begin{array}{ll}
\boldsymbol{K} & \boldsymbol{J}
\end{array}\right] \cdot \boldsymbol{T}_{6}^{H} \cdot \boldsymbol{T}_{6} \cdot\left[\begin{array}{l}
\boldsymbol{D} \\
\boldsymbol{B}
\end{array}\right]=\left[\begin{array}{ll}
\boldsymbol{M}^{*} & \boldsymbol{M}
\end{array}\right] \cdot\left[\begin{array}{c}
\boldsymbol{p} \\
\boldsymbol{p}^{*}
\end{array}\right]=\boldsymbol{m}_{N D}\left(\boldsymbol{p}(z), \boldsymbol{p}^{*}(z)\right)
$$

where $\boldsymbol{p}$ is a $1 \times 3$ complex vector.

The differential Mueller matrix clearly appears as a function of the $6 \times 1$ complex vectors:

$$
\sigma=\left[\begin{array}{c}
\boldsymbol{p} \\
\boldsymbol{p}^{*}
\end{array}\right]=\boldsymbol{T}_{6} \cdot\left[\begin{array}{l}
\boldsymbol{D} \\
\boldsymbol{B}
\end{array}\right]=\frac{1}{\sqrt{2}}\left[\begin{array}{l}
\boldsymbol{D}-i \boldsymbol{B} \\
\boldsymbol{D}+i \boldsymbol{B}
\end{array}\right]
$$

From Eq. (7) under a second order approximation hypothesis, the differential matrix $\boldsymbol{m}(z)$ is given by:

$$
\boldsymbol{m}(z)=m_{1}(z)+m_{2}(z)=\left[\boldsymbol{M}^{*} \boldsymbol{M}\right] \cdot\left[\begin{array}{c}
\boldsymbol{p} \\
\boldsymbol{p}^{*}
\end{array}\right]=\boldsymbol{m}_{N D}\left(\boldsymbol{p}(z), \boldsymbol{p}^{*}(z)\right)
$$

From Eq. (12) and the definition of first and second order cumulants [12] we have: 


$$
m_{1}(z)=\int_{0}^{z} d z_{1}\left[\begin{array}{ll}
\boldsymbol{M}^{*} & \boldsymbol{M}
\end{array}\right] \cdot\left[\begin{array}{c}
\langle\boldsymbol{p}\rangle \\
\left\langle\boldsymbol{p}^{*}\right\rangle
\end{array}\right]
$$

And

$$
m_{2}(z)=\int_{0}^{z} d z_{2} \int_{0}^{z_{2}} d z_{1}\left[\boldsymbol{M}^{*} \boldsymbol{M}\right] \operatorname{Cov}\left(\sigma\left(z_{2}\right), \sigma\left(z_{1}\right)\right)\left[\begin{array}{l}
\boldsymbol{M}^{\boldsymbol{T}} \\
\boldsymbol{M}^{* \boldsymbol{T}}
\end{array}\right]
$$

Where:

$$
\operatorname{Cov}\left(\sigma\left(z_{2}\right), \sigma\left(z_{1}\right)\right)=\left[\begin{array}{cc}
\Gamma_{p p} & C_{p p} \\
C_{p p}^{*} & \Gamma_{p p}^{*}
\end{array}\right]
$$

Stands for the covariance matrix between the complex vector $\sigma$ at $z_{1}$ and $z_{2}$ respectively with:

and:

$$
\Gamma_{p p}=\left\langle\boldsymbol{p}\left(z_{1}\right) \boldsymbol{p}^{H}\left(z_{2}\right)\right\rangle=\frac{1}{2}\left\{\Lambda_{D D}\left(z_{1}, z_{2}\right)+\Lambda_{B B}\left(z_{1}, z_{2}\right)+i .\left[\Lambda_{D B}\left(z_{1}, z_{2}\right)-\Lambda_{B D}\left(z_{1}, z_{2}\right)\right]\right\}
$$

$$
\boldsymbol{C}_{p p}=\left\langle\boldsymbol{p}\left(z_{1}\right) \boldsymbol{p}^{T}\left(z_{2}\right)\right\rangle=\frac{1}{2}\left\{\Lambda_{D D}\left(z_{1}, z_{2}\right)-\Lambda_{B B}\left(z_{1}, z_{2}\right)-i .\left[\Lambda_{D B}\left(z_{1}, z_{2}\right)+\Lambda_{B D}\left(z_{1}, z_{2}\right)\right]\right\}
$$

$\Lambda_{X Y}\left(z_{1}, z_{2}\right)=\left\langle\boldsymbol{X}\left(z_{1}\right) \boldsymbol{Y}^{T}\left(z_{2}\right)\right\rangle$ in Eqs. (18) and (19) is the classical covariance matrix between a real vector $\boldsymbol{X}$ at $z_{1}$ and a real vector $\boldsymbol{Y}$ at $z_{2}$.

It is worth noticing that describing the second-order behavior of complex variable (or process) $\boldsymbol{p}$ needs the knowledge of $\Gamma_{p p}$ and $\boldsymbol{C}_{p p}$

$\Gamma_{p p}$ is the classical covariance matrix between the complex vector $\boldsymbol{p}$ at $z_{1}$ and $z_{2}$ respectively and $\boldsymbol{C}_{p p}$ is commonly referred to under the name of complementary covariance matrix [18].

With Eq. (17) and the conjugation property between $\boldsymbol{N}$ and $\boldsymbol{M}$, Eqs. (15) - (16) become respectively:

$$
\left\{\begin{array}{c}
\boldsymbol{m}_{1}(z)=\int_{0}^{z} d z_{1}\left[\Delta_{1}(z)+\Delta_{1}^{*}(z)\right] \\
\boldsymbol{m}_{2}(z)=\int_{0}^{z} d z_{2} \int_{0}^{z_{2}} d z_{1}\left[\left(\Delta_{2}(z)+\Delta_{2}^{*}(z)\right)\right]
\end{array}\right.
$$

with

$$
\left\{\begin{array}{c}
\Delta_{1}(z)=\left(M^{*} \cdot\langle p\rangle\right) \\
\Delta_{2}(z)=N \Gamma_{p p} M^{T}+N C_{p p} N^{T}
\end{array}\right.
$$

$\boldsymbol{N} \Gamma_{p p} \boldsymbol{M}^{\boldsymbol{T}}$ is termed [14] the complex cumulant of order (1,1) and $\boldsymbol{N} \boldsymbol{C}_{p p} \boldsymbol{N}^{\boldsymbol{T}}$ is the complex cumulant of order $(2,0)$.

Using

- on the one hand the decomposition of the differential Mueller matrix proposed by Ossikovski [19] into its nondepolarizing and depolarizing part (verifying the Minkowski antisymmetric and symmetric property respectively),

- on the other hand the commutation property of $\boldsymbol{M}_{i}$ et $\boldsymbol{N}_{j}$ matrices, is straightforward to verify that:

- $\boldsymbol{m}_{1}(z)$ gives a non-depolarizing contribution

- $\left[\Delta_{1}(z), \Delta_{1}^{*}(z)\right]=0$ (the $\Delta_{1}$ matrix and its conjugate commutes)

Decomposing $\boldsymbol{m}_{1}(z)$ according to Eq. (20) is thus exactly the decomposition of non-depolarizing differential Mueller matrix according to the $\boldsymbol{Z}$ matrix or state-generating matrix recently introduced by Kuntman [11] and also analyzed by Sheppard [10]. 


\subsection{State-generating matrix for depolarizing differential Mueller matrix}

Let us now consider the decomposition of $\boldsymbol{m}_{2}(z)$. In the general case, this second order term breaks down into a depolarizing contribution and a non-depolarizing one. The existence of this latter contribution can be related to the statistical properties of the underlying processes.

From the standpoint of statistical processes it is therefore justified to regard the complex processes $L, L^{\prime}$ and $C$ of Eq. (5) and their conjugates simultaneously. From Eq. (14) it is equivalent to consider the corresponding $\Gamma_{p p}$ and $\boldsymbol{C}_{p p}$ at least in a second-order approach.

Considering this new parametrization is nevertheless more interesting for analyzing the depolarizing and nondepolarizing effects present in $\boldsymbol{m}_{2}(z)$.

We first introduce the following notation for the covariance matrices:

$$
\Lambda_{X Y}\left(z_{1}, z_{2}\right)=\left\langle\boldsymbol{X}\left(z_{1}\right) \boldsymbol{Y}^{T}\left(z_{2}\right)\right\rangle=\left[\begin{array}{lll}
x 1 y 1 & x 1 y 2 & x 1 y 3 \\
x 2 y 1 & x 2 y 2 & x 2 y 3 \\
x 3 y 1 & x 3 y 2 & x 3 y 3
\end{array}\right]
$$

Where $\boldsymbol{X}, \boldsymbol{Y}=\mathbf{D}, \mathbf{B}$ and the convention that $x i$ is evaluated at $z_{1}$ and $y j$ at $z_{2}$.

The general expression of $\boldsymbol{N} \Gamma_{p p} \boldsymbol{M}^{\boldsymbol{T}}+\boldsymbol{N} \boldsymbol{C}_{p p} \boldsymbol{N}^{\boldsymbol{T}}$ is given in Appendix A using this new notation. It is worth noticing that the cross-correlation between same nature (linear, $45^{\circ}$-linear or circular) birefringence and dichroism terms only appear on imaginary part of the diagonal entries of $\boldsymbol{\Delta}_{2}(z)$ matrix (see Eq. (A1)). Since $\boldsymbol{m}_{2}(z)$ is the summation of $\boldsymbol{\Delta}_{2}(z)$ with its conjugate, all these cross-correlations are not preserved in the $\boldsymbol{m}_{2}(z)$ expression. We find the same phenomenon as for the global phase in the non-depolarizing case as described by Kuntman [11].

Obviously the fact that this information is not explicitly allowed in $\boldsymbol{m}_{2}(z)$ does not mean that it does not affect the depolarizing behavior of the medium.

An example of this phenomenon can be found in [15], where the existence of a correlation between birefringence and dichroism terms causes the appearance of damped oscillations in the depolarization curve.

It is well known [20] that the different configurations of the $\boldsymbol{C}_{p p}$ term lead to different statistical properties of the associated complex processes. These different configurations for $\boldsymbol{C}_{p p}$ can be straightforwardly related to the existence of a non-depolarizing contribution coming from the second-order terms which adds to the non-depolarizing term coming from the mean values of the processes given by Eq. (15).

According to the different nature of the complementary covariance matrix, $\operatorname{MAP}\left(\boldsymbol{m}_{2}(z)\right)=\frac{1}{2}\left[\boldsymbol{m}_{2}(z)-\right.$ $\left.\boldsymbol{G} \cdot \boldsymbol{m}_{2}^{T}(z), \boldsymbol{G}\right]$ the Minkowski Antisymmetric Part of $\boldsymbol{m}_{2}(z)$ (with $\boldsymbol{G}=\operatorname{diag}(1,-1,-1,-1)$ ) takes the following expressions:

If $\boldsymbol{C}_{p p}$ is real:

$\Lambda_{D B}\left(z_{1}, z_{2}\right)=-\Lambda_{B D}\left(z_{1}, z_{2}\right)$ gives :

$$
M A P\left(\boldsymbol{m}_{2}(z)\right)=\left[\begin{array}{cccc}
0 & 0 & 0 & 0 \\
0 & 0 & \Delta C B & \Delta L B^{\prime} \\
0 & -\Delta C B & 0 & -\Delta L B \\
0 & -\Delta L B^{\prime} & \Delta L B & 0
\end{array}\right]
$$

With:

$$
\begin{aligned}
\Delta C B & =\frac{d 1 d 2-d 2 d 1+b 2 b 1-b 1 b 2}{2} \\
\Delta L B & =\frac{d 3 d 2-d 2 d 3+b 2 b 3-b 3 b 2}{2}
\end{aligned}
$$




$$
\Delta L B^{\prime}=\frac{d 1 d 3-d 3 d 1+b 3 b 1-b 1 b 3}{2}
$$

A non-depolarizing contribution is generated by the second order cumulant with birefringence terms related to the existence of correlations between the different components of dichroism vector or birefringence vector respectively.

If $\boldsymbol{C}_{p p}$ is purely imaginary:

$\Lambda_{D D}\left(z_{1}, z_{2}\right)=\Lambda_{B B}\left(z_{1}, z_{2}\right)$ gives:

$$
\operatorname{MAP}\left(\boldsymbol{m}_{2}(z)\right)=\left[\begin{array}{cccc}
0 & -\Delta L D & -\Delta L D^{\prime} & \Delta C D \\
-\Delta L D & 0 & 0 & 0 \\
-\Delta L D^{\prime} & 0 & 0 & 0 \\
\Delta C D & 0 & 0 & 0
\end{array}\right]
$$

with:

$$
\begin{aligned}
\Delta L D & =\frac{d 2 b 3-b 3 d 2+b 2 d 3-d 3 b 2}{2} \\
\Delta L D^{\prime} & =\frac{d 3 b 1-b 1 d 3+b 3 d 1-d 1 b 3}{2} \\
\triangle C D & =\frac{d 2 b 1-b 1 d 2+b 2 d 1-d 1 b 2}{2}
\end{aligned}
$$

A non-depolarizing contribution is generated by the second order cumulant with dichroism terms related to the existence of correlations between the different components of dichroism vector and birefringence vector.

These effects of correlation are very similar to artifacts reported by Jansen [4] with the modulation techniques in polarization spectroscopy. With this method of measurement, the artifacts result from coupling of instrumental birefringence or dichroism with birefringence or dichroism of the sample. This combined effect on light from artifact and sample is given through a matrix product between the Mueller matrix corresponding to the artefacts and the Mueller matrix of the sample. Jansen noted for instance that circular dichroism of the sample may thereby couple with linear birefringence of the instrument to give a signal interpreted as linear dichroism.

Similarly, as a second order terms, the considered cumulants (or correlation terms) lead to matrix products between matrices associated to the dichroism vector and the birefringence vector.

Eq. (26) and Eq. (24) show how the non-depolarizing contributions are generated by the second order cumulants and how ultimately the existence of a depolarization effect can affect the knowledge of the non-depolarizing part of the Mueller matrix of such a medium.

If $\boldsymbol{C}_{p p}=0 \operatorname{MAP}\left(\boldsymbol{m}_{2}(z)\right)=0$ and no supplementary contribution from the second-order terms is added to the nondepolarizing term coming from the mean values of the processes.

Obviously in the case where $\boldsymbol{C}_{p p}$ is complex, we find both the previous contributions of birefringence and dichroism.

It is therefore straightforward to decompose the non-depolarizing $\left(\boldsymbol{m}_{N D}\right)$ and depolarizing $\left(\boldsymbol{m}_{D}\right)$ contribution of the differential Mueller matrix [19] into complex conjugate matrices:

$$
\left\{\begin{array}{c}
\boldsymbol{m}_{N D}(z)=\int_{0}^{z} d z_{1}\left[\Delta_{N D}(z)+\Delta_{N D}^{*}(z)\right] \\
\boldsymbol{m}_{D}(z)=\int_{0}^{z} d z_{2} \int_{0}^{z_{2}} d z_{1}\left[\left(\Delta_{D}(z)+\Delta_{D}^{*}(z)\right)\right]
\end{array}\right.
$$

With: 


$$
\left\{\begin{array}{c}
\Delta_{N D}(z)=\Delta_{1}(z)+M A P\left[\Delta_{2}(z)\right] \\
\Delta_{D}(z)=\operatorname{MSP}\left[\Delta_{2}(z)\right]
\end{array}\right.
$$

Where $\operatorname{MSP}(\boldsymbol{X})=\frac{1}{2}\left[\boldsymbol{X}+\boldsymbol{G} \cdot \boldsymbol{X}^{\boldsymbol{T}} \cdot \boldsymbol{G}\right]$ stands for the Minkowski Symmetric Part of $\boldsymbol{X}$.

The decomposition of $\boldsymbol{m}_{D}(z)$ can be regarded as the corresponding $\boldsymbol{Z}$ matrix or state-generating matrix for the depolarizing term of the differential Mueller matrix.

It is worth noticing that $\Delta_{N D}(z)$ and $\Delta_{N D}^{*}(z)$ do not commute now without supplementary hypothesis. Upon substituting Eq. (18), Eq. (19) and Eq. (21) into the computation of Eq. (29), it is also possible to verify that the matrices $\Delta_{D}(z)$ and $\Delta_{D}^{*}(z)$ do not commute.

\subsection{Stationnarity and parity hypothesis.}

With a second order stationary hypothesis for the processes - meaning that $\Lambda_{X Y}\left(z_{1}, z_{2}\right)=\Lambda_{X Y}\left(z_{2}-z_{1}\right)=\Lambda_{X Y}(\tau)-$ Eq. (18) and Eq. (19) become:

$$
\left\{\begin{array}{l}
\Gamma_{p p}(\tau)=\frac{1}{2}\left\{\Lambda_{D D}(\tau)+\Lambda_{B B}(\tau)+i \cdot\left[\Lambda_{B D}^{T}(-\tau)-\Lambda_{B D}(\tau)\right]\right\} \\
\boldsymbol{C}_{p p}(\tau)=\frac{1}{2}\left\{\Lambda_{D D}(\tau)-\Lambda_{B B}(\tau)-i \cdot\left[\Lambda_{B D}^{T}(-\tau)+\Lambda_{B D}(\tau)\right]\right\}
\end{array}\right.
$$

Under a stationnarity hypothesis and a parity hypothesis (being even) for the correlation functions, it is straightforward to demonstrate (see Eq. (24) and Eq. (2628) ) that the supplementary contribution from the secondorder terms to the non-depolarizing term vanishes and we have:

$$
\begin{gathered}
M A P\left[\Delta_{2}(z)\right]=0 \\
\Delta_{D}(z)=\Delta_{2}(z)=\boldsymbol{N} \Gamma_{p p} M^{T}+N C_{p p} \boldsymbol{N}^{T}
\end{gathered}
$$

It is worth noticing that in this case $\boldsymbol{N} \boldsymbol{C}_{p p} \boldsymbol{N}^{\boldsymbol{T}}$ the complex cumulant of order $(2,0)$ (its symmetric $\left(\boldsymbol{N} \boldsymbol{C}_{p p} \boldsymbol{N}^{\boldsymbol{T}}\right)^{*}$ the cumulant of order $(0,2)$ respectively) has a very simple expression (complex conjugate expression respectively) given by:

$$
[d 1 d 1+d 2 d 2+d 3 d 3-I(b 1 d 1+b 2 d 2+b 3 d 3)] . I_{4}
$$

Where $\boldsymbol{I}_{4}$ stands for the identity matrix of order 4 . So, on one hand, all the correlation terms contribute as an isotropic absorption, and the orher hand, the cross-correlations contribute as a global phase but this global phase information is not allowed in $\boldsymbol{m}(z)$. Both these terms evolve as $z$-functions.

An example of such hypothesis (stationnarity and parity hypothesis) is found for instance in [13] for homogeneous medium where a zero order approximation of the correlation functions is made. This zero order approximation means that the correlation is assumed to be locally constant and is valid on a much greater length than the amplitude of the fluctuations is low since a sufficient decay has to be ensured by the condition $\alpha . \zeta \ll 1$ (where $\alpha$ formally denotes the amplitude of the fluctuations and $\zeta$ the correlation distance) to have vanishing higher orders of the correlation function (see [12] for details).

Such hypothesis are then valid for very thin samples or for thicker samples but with smaller fluctuations (thus a lower depolarization since the level of depolarization is obviously a function of the amplitude of the fluctuations but in a nontrivial way) and as long as it can be considered that the correlation is constant.

We then have the following relations between the covariance matrices: 


$$
\Lambda_{D B}(\tau)=\Lambda_{B D}^{T}(-\tau)=\Lambda_{B D}^{T}(\tau)=\Lambda_{B D}^{T}(0)
$$

Which leads to a general expression of $\boldsymbol{\Delta}_{D}(z)$ given in Appendix $\boldsymbol{B}$.

In the rest of this article $\Lambda_{D B}(\tau)$ and $\Lambda_{B D}(\tau)$ respectively, will be denoted by $\Lambda_{D B}$ and $\Lambda_{B D}$ respectively since in this case the covariance matrices no longer depend on the $\tau$ variable. This result leads to a $z$-quadratic solution $(z$ is the thickness of the medium) for the depolarizing part of the differential matrix obtained as double integration of constant terms (see Eq.(16)).

In the same way, stationarity imposes constant mean values. This result leads to a $z$-linear solution for the nondepolarizing part of the differential matrix. It is worth noticing that this $z$-linear solution for the non-depolarizing differential Mueller matrix is not necessarily valid if the processes are not stationary since a contribution of second order is added to the first order cumulant contribution.

Eventually, if an approximation to the zero order of the correlation functions of the processes - that are then stationary - is assumed, it is possible to verify that the matrices $\Delta_{N D}(z)=\Delta_{1}(z)$ and $\Delta_{N D}^{*}(z)$ commute and it is also possible to verify that the matrices $\Delta_{D}(z)=\Delta_{2}(z)$ and $\Delta_{D}^{*}(z)$ commute.

On the other hand, it is not possible to hope that $\Delta_{N D}(z)+\Delta_{D}(z)$ and its conjugate commute in the general case because it would require that $\boldsymbol{m}_{N D}(z)$ and $\boldsymbol{m}_{D}(z)$ commute what is wrong most of the time.

Decomposing the differential Mueller matrix according to the $\boldsymbol{Z}$ matrix recently introduced by Kuntman [11] is therefore possible for depolarizing medium at least in the second order approximation with some hypothesis on the statistical properties of the processes. We then have $\boldsymbol{Z}_{D}(z)=\exp \left[\Delta_{D}(z)\right]$ with the expression of $\Delta_{D}(z)=\Delta_{2}(z)$ given by Eq. (21).

\section{SCALAR COMPLEX VARIABLE CASE.}

Let us considering the case where only one of the three components of the spectroscopic complex vector $\left(L, L^{\prime}, C\right)$ is operative. For example, let $L$ be this component (this choice is obviously not restrictive). We then have a scalar complex variable that we assume Gaussian for example.

Fig. 1 shows an example of probability density contour when $L$ component is the operative one. It is usual to introduce the circularity quotient [20] as a complex coefficient defined by:

$$
\rho=\frac{C_{L L}}{\Gamma_{L L}}=\frac{\operatorname{Var}(L D)-\operatorname{Var}(L B)+2 \cdot i \cdot \operatorname{Cov}(L D, L B)}{\operatorname{Var}(L D)+\operatorname{Var}(L B)}
$$

Where $\operatorname{Var}(X)-\operatorname{Cov}(X, Y)$ respectively - stands for the variance of $X$ - the covariance of $X$ and $Y$ respectively -. We will also use classical notation $\operatorname{Var}(X)=\sigma_{X}^{2}$

This circularity quotient with a polar representation is given by $\rho=r \cdot \exp (j \psi)$ where the quantity $\psi$ is termed the circularity angle of $L$ and $r$ is the square of the ellipse eccentricity.

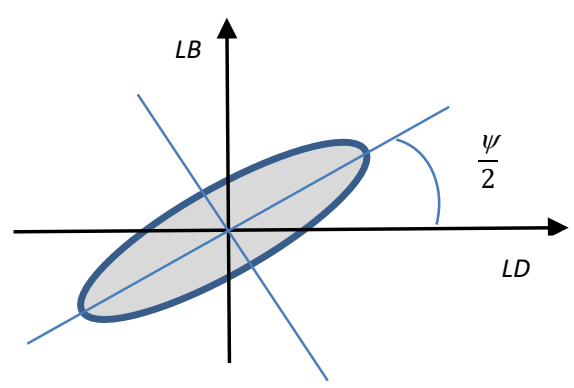


Fig. 1. Probability density contours of the scalar complex Gaussian variable $L$.

From equations (33) the case $\psi=0$ corresponds to $d 1 b 1=0$, meaning no correlation between the $L B$ and $L D$ components.

But it is obvious that the value of $\psi$ depends on the position adopted for the axes $L B$ and $L D$. A rotation of the axes of an angle $\psi / 2$ thus makes it possible to cancel $\psi$, i.e. to de-correlate the components. It is therefore the adopted system of representation that creates this "apparent" correlation between the physical quantities concerned.

Nevertheless this is of no consequence for the depolarization measurement. Indeed when $L$ is the only operative component, it is possible [12] [15] to show that:

$$
m_{D}=\operatorname{diag}\left(1,0,-\sigma_{L}^{2},-\sigma_{L}^{2}\right)
$$

where $\sigma_{L}^{2}=E\left(|L|^{2}\right)=\sigma_{L D}^{2}+\sigma_{L B}^{2}$.

The $L D$ and $L B$ variances can be expressed [20] as:

$$
\left\{\begin{array}{l}
d 1 d 1=\sigma_{L D}^{2}=\lambda_{1} \cos ^{2}(\alpha)+\lambda_{2} \sin ^{2}(\alpha) \\
b 1 b 1=\sigma_{L B}^{2}=\lambda_{1} \sin ^{2}(\alpha)+\lambda_{2} \cos ^{2}(\alpha)
\end{array}\right.
$$

with $\alpha=\frac{\psi}{2}$ and $\lambda_{1}, \lambda_{2}$ stand for the eigenvalues of the $2 \times 2$ real covariance matrix associated to $[L D L B]^{\mathrm{T}}$ random real vector. From Eq. (35) it is obvious to see that:

$$
\sigma_{L}^{2}=\lambda_{1}+\lambda_{2}
$$

This result shows that $\psi$ (this "apparent" correlation) is of no consequence for the depolarization measurement in an incoherent wave configuration.

On the other hand, the global phase - see Eq. (31) - related to the $d 1 b 1$ term (correlation between $L B$ and $L D$ terms) evolves as quadratic function of $z$ - see Eq. (27) - and is related to the $\psi$ value, meaning the laboratory $x$-y axes definition of the measure. The existence of this global phase must be taken into account when processing coherent waves.

\section{CONCLUSION}

In this work, we have shown how the information contained in differentiel Mueller matrix related to a depolarizing medium can be expressed in terms of a state-generating matrix. The formulation of the problem using complex random vectors makes it possible to directly introduce the formalism of state-generating matrix in the case of differential depolarizing matrices.

Under a second order approximation hypothesis, this depolarizing state-generating matrix is directly related not only to the classical covariance matrix of the associated complex vector but also to the complementary covariance matrix. The different configurations (null, imaginary, real) for this complementary covariance matrix can be straightforwardly associated to physical consequences.

The commuting property of these state-generating matrices obtained in the case of homogeneous non-depolarizing media is preserved in the case where these media become depolarizing if an approximation to the zero order of the correlation functions of the processes are made. Such hypothesis are then valid only for very thin samples or for thicker samples but with very low depolarization. 
Eventually, examples of physical interpretations that can be obtained have been presented specifically for homogeneous medium. Illustrations are given when the complex vector degenerates into a complex scalar and when a Gaussian random processes hypothesis is made.

\section{APPENDIX A}

From Eq. (18)-(19) and the definition of $\mathrm{M}$ and N, it is straightforward to show that:

$$
N \Gamma_{p p} M^{T}+N C_{p p} N^{T}=\frac{1}{2}\left(\delta_{i j}\right)
$$

with:

$$
\begin{aligned}
& \delta_{11}=d 1 d 1+d 2 d 2+d 3 d 3-I(b 1 d 1+b 2 d 2+b 3 d 3) \\
& \delta_{12}=d 3 b 2-d 2 b 3+I(b 2 b 3-b 3 b 2) \\
& \delta_{13}=d 1 b 3-d 3 b 1+I(b 3 b 1-b 1 b 3) \\
& \delta_{14}=d 2 b 1-d 1 b 2+I(b 1 b 2-b 2 b 1) \\
& \delta_{22}=d 1 d 1-b 2 b 2-b 3 b 3-I(b 1 d 1+d 2 b 2+d 3 b 3) \\
& \delta_{21}=b 3 d 2-b 2 d 3+I(d 3 d 2-d 2 d 3) \\
& \delta_{23}=d 1 d 2+b 2 b 1+I(d 2 b 1-b 1 d 2) \\
& \delta_{24}=d 1 d 3+b 3 b 1+I(d 3 b 1-b 1 d 3) \\
& \delta_{33}=d 2 d 2-b 1 b 1-b 3 b 3-I(d 1 b 1+b 2 d 2+d 3 b 3) \\
& \delta_{31}=b 1 d 3-b 3 d 1+I(d 1 d 3-d 3 d 1) \\
& \delta_{32}=d 2 d 1+b 1 b 2+I(d 1 b 2-b 2 d 1) \\
& \delta_{34}=d 2 d 3+b 3 b 2+I(d 3 b 2-b 2 d 3) \\
& \delta_{44}=d 3 d 3-b 1 b 1-b 2 b 2-I(d 1 b 1+d 2 b 2+b 3 d 3) \\
& \delta_{41}=b 2 d 1-b 1 d 2+I(d 2 d 1-d 1 d 2) \\
& \delta_{42}=d 3 d 1+b 1 b 3+I(d 1 b 3-b 3 d 1) \\
& \delta_{43}=d 3 d 2+b 2 b 3+I(d 2 b 3-b 3 d 2)
\end{aligned}
$$

\section{APPENDIX B}

Expression og $\Delta_{D}(z)$ for homogeneous medium where a zero order approximation of the correlation functions is assumed.:

$$
\begin{gathered}
\Delta_{D}(z)=\frac{1}{2}\left(\delta_{i j}\right) \\
\delta_{11}=d 1 d 1+d 2 d 2+d 3 d 3-I(b 1 d 1+b 2 d 2+b 3 d 3) \\
\delta_{12}=d 3 b 2-d 2 b 3 \\
\delta_{13}=d 1 b 3-d 3 b 1 \\
\delta_{14}=d 2 b 1-d 1 b 2 \\
\delta_{22}=d 1 d 1-b 2 b 2-b 3 b 3-I(b 1 d 1+d 2 b 2+d 3 b 3) \\
\delta_{21}=b 3 d 2-b 2 d 3 \\
\delta_{23}=d 1 d 2+b 2 b 1 \\
\delta_{24}=d 1 d 3+b 3 b 1 \\
\delta_{33}=d 2 d 2-b 1 b 1-b 3 b 3-I(d 1 b 1+b 2 d 2+d 3 b 3) \\
\delta_{31}=b 1 d 3-b 3 d 1
\end{gathered}
$$




$$
\begin{gathered}
\delta_{32}=d 2 d 1+b 1 b 2 \\
\delta_{34}=d 2 d 3+b 3 b 2 \\
\delta_{44}=d 3 d 3-b 1 b 1-b 2 b 2-I(d 1 b 1+d 2 b 2+b 3 d 3) \\
\delta_{41}=b 2 d 1-b 1 d 2 \\
\delta_{42}=d 3 d 1+b 1 b 3 \\
\delta_{43}=d 3 d 2+b 2 b 3
\end{gathered}
$$

\section{References}

1. R.C. Jones, "A new calculus for the treatment of optical systems. VII properties of the N-matrices," J. Opt. Soc. Am. 38, 671-685 (1948)

2. N. Go, " Optical Activity of Anisotropic Solutions, " J. Phys. Soc. Jpn. 23, 88-97 (1967)

3. R. M. A. Azzam, "Propagation of partially polarized light through anisotropic media with or without depolarization: A differential 4x4 matrix calculus," J. Opt. Soc. Am. 68, 1756-1767 (1978)

4. H.P. Jensen, J.A. Schellman, T. Troxell, "Modulation techniques in polarization spectroscopy," Appl. Spectroscopy 32, $192-200$ (1978)

5. O. Arteaga and B. Kahr, "Characterization of homogenous depolarizing media based on Mueller matrix differential decomposition," Opt. Lett. 38, 1134-1136 (2013).

6. H. J. Hofrichter and J. Schellman in Conformation of Biological Molecules and Polymers, Jerusalem Symposia on Quantum Chemistry and Biochemistry V, pp 787-807, Israel Academy of Sciences and humanities, Jerusalem, 1973.

7. J. Schellman, H.P. Jensen, "Optical spectroscopie of oriented molecules," Chem. Rev. 87, 1359-1399 (1987)

8. V. Devlaminck, "Physical model of differential Mueller matrix for depolarizing uniform media," J. Opt. Soc. Am. 30, 2196-2204 (2013)

9. Wu-Ki Tung, Group Theory in Physics (World Scientific Publishing, Philadelphia Singapore, 1985)

10. C. Sheppard, A. Bendandi, A. Le Gratiet, and A. Diaspro, "Coherency and differential Mueller matrices for polarizing media," J. Opt. Soc. Am. A 35, 2058-2069 (2018)

11. E. Kuntman, M. Ali Kuntman, and O. Arteaga, "Vector and matrix states for Mueller matrices of nondepolarizing optical media," J. Opt. Soc. Am. A 34, 80-86 (2017)

12. V. Devlaminck, "Depolarizing differential Mueller matrix of homogeneous media under Gaussian fluctuation hypothesis," J. Opt. Soc. Am. A 32, 1736-1743 (2015).

13. R. Ossikovski, O. Arteaga, "Statistical meaning of the differential Mueller matrix of depolarizing homogeneous media," Opt. Letters. 39, 4470-4473 (2014)

14. P.O. Amblard, M. Gaeta, J.L. Lacoume, "Statistics for complex variables and signals," Signal Processing, 53, 1-25 (1996)

15. J. Charbois and V. Devlaminck, "Stochastic model for the differential Mueller matrix of stationary and nonstationary turbid media," J. Opt. Soc. Am. A 33, 2414-2424 (2016).

16. R.F. Fox, "Gaussian stochastic processes in physics," Phy. Rep. 48, 179-283 (1978)

17. A. van den Bos, "The multivariate complex normal distribution- A generalization," IEEE Trans. Inform. Theory 41, 537539 (1995)

18. P.J. Schreier, L.L. Scharf, "Second-order analysis of improper complex random vectors and processes," IEEE Trans. on Signal Processing, 51, 714-725 (2003)

19. R. Ossikovski, "Differential matrix formalism for depolarizing anisotropic media," Opt. Letters. 36, 2330-2332 (2011)

20. E. Ollila,"On the circularity of complex random variable," IEEE Signal Processing Lett. 15, 841-844 (2008) 
